MATEC Web of Conferences 22,05026 (2015)

DOI: $10.1051 /$ matec conf/ 20152205026

(C) Owned by the authors, published by EDP Sciences, 2015

\title{
A Study on Aging Characteristics of Silicone Oil in HV Oil-filled Cable Termination Based on Infrared Thermal Imaging Test
}

\author{
Zhiyong Chen \& Jie Tang \\ Electric Power Research Institute of Guangxi Power Grid CO., LTD., Nanning, Guangxi, China \\ Chuanxian Luo \& Jing Zhang \\ State Grid Electric Power Research Institute Wuhan NARI CO., LTD., Wuhan, Hubei, China
}

\begin{abstract}
In order to assess the aging characteristics of Silicone oil in the high voltage oil-filled cable termination after being operated 12 years, the silicone oil in service were studied using the infrared imaging detection, Fourier infrared spectrum analysis, and dielectric properties and micro water content test. The relationship between the infrared thermal imaging temperature and the physical and chemical properties was considered. The results indicate that, the operating temperature of silicone oil within the terminal based on the infrared thermal imaging testing can be characterization of silicone oil aging state. The aging characteristic peak of Silicone oil is $\mathrm{P}-\mathrm{H}$ key stretching vibration peak, which is near $2360 \mathrm{~cm}-1$, Si-CH3, $-\mathrm{CH} 3$ and Si-O-Si keys have no influence of before and after aging. In addition, the aging properties of silicone oil can be reviewed by the change of the dielectric properties, and the decline of the dielectric properties is caused by the aging and water content of materials. The results will be helpful for research of silicone oil aging and optimization design of anti-aging, serving as references for the manufacture, design, and operation.
\end{abstract}

Keywords: HV oil-filled cable terminal; silicone oil; aging; the infrared thermal imaging; the dielectric properties

\section{INTRODUCTION}

High voltage cable termination is an important accessory to connect to other electrical equipment when high voltage cable lying. According to the structure and usages, it can be divided into many types, such as oil-filled cable termination, dry-type cable termination, GIS termination, outdoor cable termination and indoor cable termination ${ }^{[1]}$. For various types of cable terminations, silicone oil-filled cable termination is widely used, because it has a mature manufacturing process and the operating experience of many years. Research relevant characteristics silicone has been a hot topic for scholars ${ }^{[2-6]}$. However, during the operation of the cable line, electrical properties of the silicone oil within the termination are aging. The aging is due to oxygen, humidity, high temperature, a strong electric field and impurities and other external factors. The cable terminal runs longer, and then the aging degree of silicone oil will gradually increase. It will result in greatly reduced insulating properties of the silicone oil, thereby causing heating of the cable termination and dielectric breakdown ${ }^{[7-12]}$.

CIGRE B1 Working Group conducted a number of fault statistics for the period 2000-2005, the voltage level of $51 \mathrm{kV}-400 \mathrm{kV}$ cable termination ${ }^{[13]}$. The results showed that: there were 61 cable terminal failures, with performance degradation caused by the failure of the insulating oil. It is obvious that insulation properties of silicone oil within termination plays a very crucial role for the safe operation of the termination.
In China, Infrared imaging detection is an important method detecting the high-voltage (HV) power equipment running state ${ }^{[14-21]}$. It is a kind of non-contact on-line measurement that can determine the HV power equipment running state, and find the fault position and predictive its future state. In the process of the infrared images analysis, the computer captures remote equipment's images, calculates images' moment invariants as characteristic vector of recognition, and recognizes power equipment by support vector machine (SVM). The system further analyzes images and if we find images have convex hull, intensive stochastic noise, or false edges, then we sequently make a conclusion whether the running state of equipment is in order ${ }^{[22]}$. In 2006, Guangzhou Power Supply Bureau detected 114 running cable terminations, using infrared thermal imaging device, and it was found to have abnormal heating phenomenon with 83 terminals. The abnormal heating cable terminations were out of operation and disintegration, and some serious insulation problems in the stress cone parts are observed. According to different fever degrees, the silicone oil was replaced, and hidden faults were eliminated ${ }^{[23]}$.

\section{EXPERIMENTAL MATERIALS AND PROCE- DURE}

\subsection{CABLE TERMINATION FEVER}

The study was performed using FLIR P630 infrared camera, and described silicone oil in the silicone rub- 
ber composite housing $220 \mathrm{kV}$ cable termination, which has been running for 12 years on site. From infrared imaging test results in Figure 1 and Table 1, the temperature distribution of the LI01 termination shows high temperatures, especially at the bottom of cable termination, the temperature difference is $7.1^{\circ} \mathrm{C}$ between the maximum temperature and the minimum temperature. The LI01 maximum temperature is $4.7^{\circ} \mathrm{C}$ higher than $\mathrm{LI} 02$, and $4.5^{\circ} \mathrm{C}$ higher than $\mathrm{LI} 03$ at the same position.

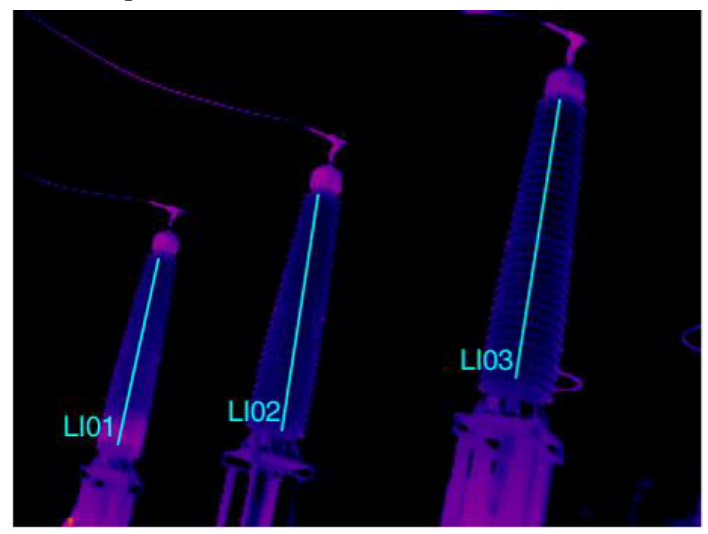

Figure 1. 220kV cable termination infrared image

Table 1. Infrared imaging data

\begin{tabular}{|l|l|l|l|}
\hline sample & $\begin{array}{c}\text { Temperature } \\
\text { Max. }{ }^{\circ} \mathrm{C}\end{array}$ & $\begin{array}{c}\text { Temperature } \\
\text { Min. }{ }^{\circ} \mathrm{C}\end{array}$ & $\begin{array}{l}\text { Temperature } \\
\text { difference } \\
{ }^{\circ} \mathrm{C}\end{array}$ \\
\hline LI01 & 26.8 & 19.7 & 7.1 \\
\hline LI02 & 21.1 & 18.2 & 3.9 \\
\hline LI03 & 21.3 & 17.8 & 3.5 \\
\hline
\end{tabular}

The materials of the work were silicone oils, which were taken out from $220 \mathrm{kV}$ oil-filled cable terminations. The oil sample is shown in Figure 2. The sample 0 \# was the same batch of purchasing silicon oil. It has been sealed for 12 years. The $1 \#$ was silicone inside the LI01 cable termination. The 2\# was silicone inside the LI02 cable termination. The $3 \#$ was silicone inside the LI03 cable termination. Figure 1 shows that, samples \#0, \#1 and \#2 were relatively clear, but sample \#3 was translucent, and there was white floc at bottom of sample \#3.

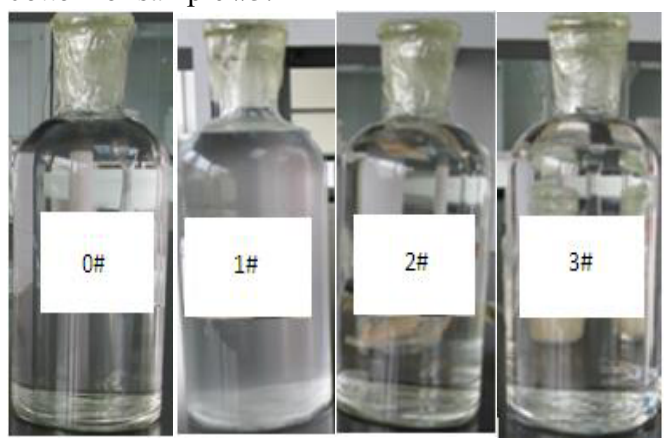

Figure 2. Silicone oil samples

\subsection{INSULATION TESTS OF SILICONE OIL}

Parameters on the electrical properties of the silicone oil sample (dielectric constant, loss tangent, volume resistivity, and breakdown strength), infrared spectrum and moisture content were measured. The standards and test equipment is shown in Table 2. The sample container is thoroughly cleaned with alcohol before testing and replacement sample. Each test was performed three times at least, and the breakdown performance was performed six times.

Table 2. Test equipment and standard

\begin{tabular}{|c|c|c|}
\hline Type & standard & equipment \\
\hline $\begin{array}{l}\text { Dielectric constant } \\
\text { and loss tangent }\end{array}$ & GB/T 5654-85 & Schering bridge. 2821 \\
\hline Volume resistivity & GB/T 5654-85 & $\begin{array}{l}\text { ZC36 High resistance } \\
\text { meter }\end{array}$ \\
\hline $\begin{array}{l}\text { The breakdown } \\
\text { field strength }\end{array}$ & $\mathrm{GB} / \mathrm{T} 507$ & 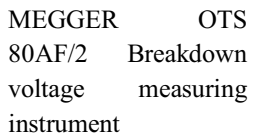 \\
\hline infrared spectrum & $\begin{array}{l}\mathrm{GB} / \mathrm{T} \\
6040-2002 \\
\end{array}$ & IRPrestige-21 \\
\hline moisture content & GB7600-1987 & $\begin{array}{l}\text { Coulomb's law of } \\
\text { trace moisture meter } \\
\text { JF-3 }\end{array}$ \\
\hline
\end{tabular}

\section{EXPERIMENTAL RESULTS}

\subsection{Infrared spectrum}

Each sample was measured using Fourier infrared spectroscopy, and the test results are shown in Figure 3. Comparing Figures 3 (a)-(d) we can see: (1) All samples was detected saturated $\mathrm{C}-\mathrm{H}$ bond $\left(-\mathrm{CH}_{3}\right)$ stretching vibration at $2962 \mathrm{~cm}^{-1}$ and $2904 \mathrm{~cm}^{-1}$, and $\mathrm{CH}$ bond $\left(-\mathrm{CH}_{3}\right)$ bending vibration peak was detected at $1444 \mathrm{~cm}-1,1413 \mathrm{~cm}^{-1}$. There were significant groups of $\mathrm{Si}-\mathrm{CH}_{3}$ absorption peak at 1260,1020 , $800 \mathrm{~cm}-1$, Si-CH3 characteristic peaks bending vibration at $1261 \mathrm{~cm}-1$; Si-O stretching vibration absorption peak at $1093 \mathrm{~cm}-1-1018 \mathrm{~cm}^{-1}$; P-O-C and P-O stretching vibration absorption peak were taken overlap at $1000-1190 \mathrm{~cm}^{-1}$; Si-C stretching vibration absorption peak at $796 \mathrm{~cm}^{-1}$. (2) Samples 0\#, 2\#, 3\# at $2380 \mathrm{~cm}^{-1}$ were detected to have a P-H stretching vibration absorption peak, while the sample \#1 P-H stretching vibration absorption peak basically has disappeared at $2380 \mathrm{~cm}^{-1}$.

Insulating oil in the cable termination was methyl silicone oil, which can be determined by infrared spectroscopy test results. In addition, it can be seen by comparing the infrared spectra of oil samples, new absorption peak did not occur in all segments, only sample 1\# P-H stretching vibration at $2380 \mathrm{~cm}-1 \mathrm{ab}-$ sorption peak receded, which means that degradation 
reaction of macromolecules containing $\mathrm{P}-\mathrm{H}$ bond had occurred, the corresponding small molecule product was generated. The level changes of absorption peak in the local area may be caused by the change in molecular weight.

\subsection{Dielectric properties}

Table 3 described the change between temperature and dielectric constant $(\varepsilon)$, the loss tangent $(\tan \delta)$, the volume resistivity $\left(\rho_{\mathrm{v}}\right)$ and breakdown field strength (E) of the sample. Compare the data in the table, dielectric constant and loss tangent values of sample $1 \#$ was larger, and then resistivity and breakdown field strength value was small. Especially when the temperature was $90^{\circ} \mathrm{C}$. Compared with $0 \#$, dielectric constant and loss tangent of $1 \#$ significantly increased, and the resistance was significantly reduced, a difference of about two orders of magnitude, 2\#, 3\# resistivity decreased about one order of magnitude. In addition, 1\#, $2 \#$ and $3 \#$ breakdown field strength was significantly lower than $0 \#$ breakdown field strength. Compared with $0 \#$, at $30^{\circ} \mathrm{C}$, sample $1 \#$ breakdown field strength decreased $41.2 \%$, sample $2 \#$ decreased $28.9 \%$, sample $3 \#$ decreased $26.4 \%$. At $90{ }^{\circ} \mathrm{C}$, sample $1 \#$ breakdown field strength decreased $56.4 \%$, sample $2 \#$ decreased $36.7 \%$, sample $3 \#$ decreased $27.3 \%$.

Table 3. Sample Dielectric Properties of Test Data

\begin{tabular}{|l|l|l|l|l|}
\hline \multirow{2}{*}{ NO. } & \multicolumn{2}{|l|}{$\boldsymbol{\rho}_{\mathbf{v}} \boldsymbol{\Omega} \cdot \mathbf{m}$} & \multicolumn{2}{l|}{$\boldsymbol{\varepsilon}$} \\
\cline { 2 - 5 } & $\mathbf{3 0} \mathrm{C}$ & $\mathbf{9 0}{ }^{\circ} \mathrm{C}$ & $\mathbf{3 0}{ }^{\circ} \mathrm{C}$ & $\mathbf{9 0}{ }^{\circ} \mathrm{C}$ \\
\hline $0 \#$ & $2.9 \times 10^{15}$ & $9.8 \times 10^{14}$ & 2.71 & 2.52 \\
\hline $1 \#$ & $2.2 \times 10^{15}$ & $5.8 \times 10^{12}$ & 2.76 & 2.69 \\
\hline $2 \#$ & $2.5 \times 10^{15}$ & $5.2 \times 10^{13}$ & 2.74 & 2.57 \\
\hline $3 \#$ & $2.6 \times 10^{15}$ & $8.1 \times 10^{13}$ & 2.73 & 2.56 \\
\hline & & & $\mathbf{E}$ & \\
\hline & $\mathbf{t a n} \boldsymbol{\delta}$ & & $\mathbf{k V} / \mathbf{m m})$ \\
\hline $0 \#$ & $1.0 \times 10^{-4}$ & $4.4 \times 10^{-4}$ & 12.72 & 10.56 \\
\hline $1 \#$ & $2.89 \times 10^{-4}$ & $8.6 \times 10^{-3}$ & 7.48 & 4.6 \\
\hline $2 \#$ & $1.7 \times 10^{-4}$ & $7.9 \times 10^{-4}$ & 9.04 & 6.68 \\
\hline $3 \#$ & $1.6 \times 10^{-4}$ & $7.3 \times 10^{-4}$ & 9.36 & 7.68 \\
\hline
\end{tabular}




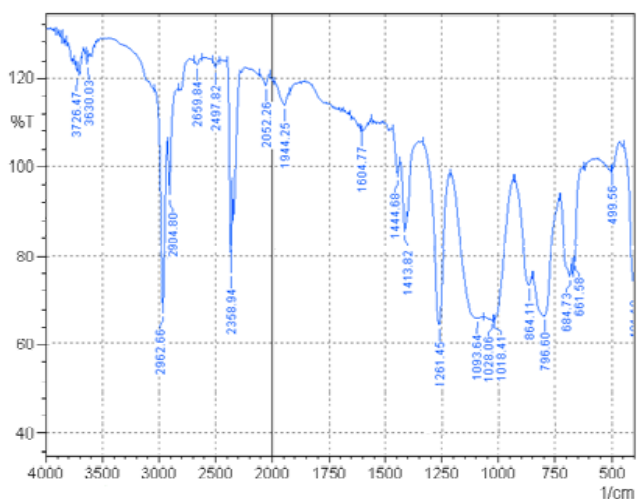

(a) $0 \#$

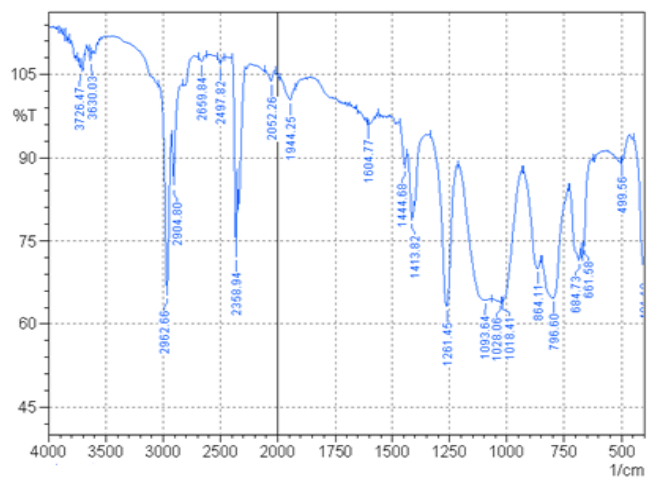

(c)2\#

Figure 3. Infrared spectra of samples

\section{DISCUSSION AND ANALYSIS}

\subsection{Infrared imaging}

Cable terminations are voltage pyrogenic type equipment ${ }^{[24]}$. Its dielectric loss is the main source equipment, because dielectric loss is proportional to the square of the voltage. Such defects are more typical in the HV and EHV cable system. Because of effect under the alternating electric field, change the polarization direction of the oil molecules, so that energy was consumed in this process, causing the cable terminal fever. The resulting heating power is characterized by the formula can be used:

$$
P=U^{2} \omega C \tan \delta
$$

Where, $\mathrm{U}$ is the applied voltage; $\omega$ is the angular frequency of the alternating voltage; $\mathrm{C}$ is the equivalent capacitance of the material; $\operatorname{tg} \delta$ is the tangent of the loss angle of the material. Accordingly, when the $\mathrm{U}$ and $\omega$ is a constant, $\mathrm{C}$ and $\operatorname{tg} \delta$ determines the silicone oil heating power in the cable terminal. The ca-

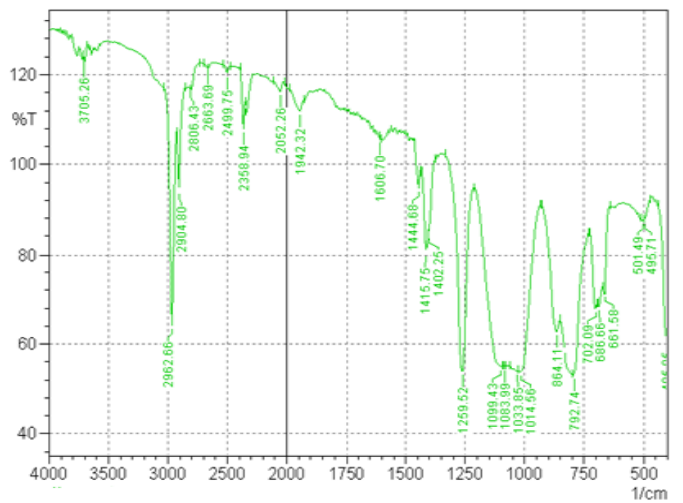

(b) $1 \#$

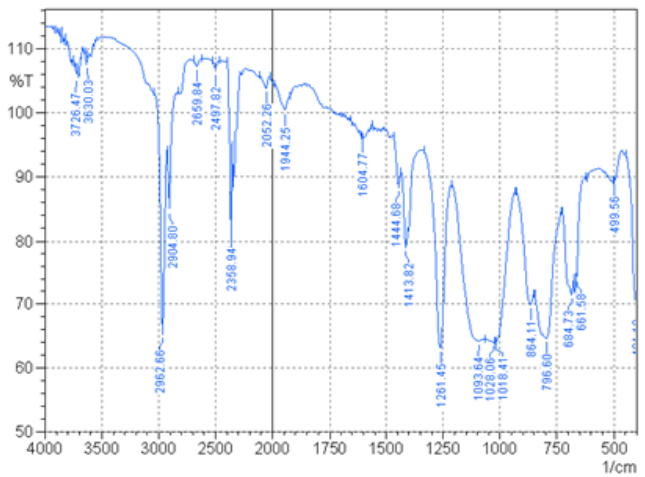

(d) $3 \#$

pacitance $\mathrm{C}$ is proportional to the dielectric constant $\varepsilon$ ${ }^{25]}$, and then the relationship is established fever macro and micro parameters. The dielectric constant of sample $1 \#$ was more high $1.85 \%$ than sample $0 \#$ at $30{ }^{\circ} \mathrm{C}$, and $6.75 \%$ at $90^{\circ} \mathrm{C}$. The loss tangent of sample $1 \#$ were $189 \%$ and $1854 \%$ higher than sample $0 \#$ at $30^{\circ} \mathrm{C}$ and $90^{\circ} \mathrm{C}$. The dielectric properties change of the high voltage oil-filled cable termination was main reasons of fever.

\subsection{Infrared spectrum}

Samples 0\#, 2\#, 3\# were P-H stretching vibration absorption peak at $2380 \mathrm{~cm}^{-1}$ by IR, but the chemical structure of methyl silicone $\mathrm{P}-\mathrm{H}$ bond did not exist, where $\mathrm{P}-\mathrm{H}$ bond was coming from is a mystery. Reference [26] pointed out that the presence of polymerization and equilibrium chemical reaction are both simultaneously during the synthesis of methyl silicone oil. Methyl silicone synthesis process is shown in Figure 4. Commonly used in the polymerization reaction $\mathrm{KOH}$ as catalyst, choose a $\mathrm{KOH}$ catalyst with less, usually from $0.0005 \%$ to $0.01 \%$ of the amount of silicone. Equilibration reactions are generally used silicone phosphate as a neutralizing agent. Silicone 
phosphate and equilibrated reaction product has excellent compatibility, and phosphate is a three protonic acid, used to form potassium dihydrogen phosphate, dipotassium hydrogen phosphate and potassium phosphate with reaction $\mathrm{KOH}$. The first two in the system may also play buffer role ${ }^{[27]}$. Thus it can be determined in IR P-H stretching vibration absorption peak due to the existence of silicone phosphate. There was not P-H stretching vibration absorption peak of the sample \#1 at $2380 \mathrm{~cm}^{-1}$, which may be due to the degradation of silicone phosphate polymer chain caused by high electric field and long-term aging.<smiles>C[Si](C)(C)O[Si](C)(C)O[Si](C)(C)O[Si](C)(C)[Si](C)(C)O[Si](C)(C)[Si](C)(C)C</smiles>

(a) Silicone oil polymerization<smiles>CC(C)([Si](C)(C)O[Si](C)(C)C)[Si](C)(C)O[Si](C)(C)C(C)(C)[Si](C)(C)O[Si](C)(C)C(C)(C)[Si](C)(C)C(C)(C)O[Si](C)(C)C</smiles>

(b) Silicone oil equilibrium reaction

Figure 4. Polymerization and equilibrium reactions chemical equation of silicone oil

\subsection{Dielectric properties}

Dielectric constant and loss tangent reflect the polarization process of the material in the electric field effect. The larger the dielectric constant and loss tangent are, the stronger the degree of polarization of the material is. Thus, it can be determined, the larger the degree of polarization of the sample \#1 is. This phenomenon may be caused by the following two aspects: (1) sample 1\# silicone oil contains large amounts of water molecules, and the water molecules are polar small molecules, more easily ionized in the electric field which is applied, so that the polarization was increased; (2) Sample 1\# in the cable termination operation for many years, silicone oil aging cracking, and macromolecular chain of polymer into smaller molecules chains, increases the dielectric constant and the loss tangent of sample $1 \#$.
Resistivity and breakdown field strength is closely related to the carrier concentration and the amount of material. The lower resistivity and breakdown field strength of sample \#1 is, described carrier (i.e., the conductive component) increase of sample, which may be due to silicone oil cracking of material from moisture and aging. The resistivity and the breakdown field strength of sample \#1 is low, that indicating sample 1\# appeared with aging also.

By analyzing the dielectric properties of all samples, we can conclude that sample $1 \#$ material has aged preliminarily. To further determine the causes of aging, we tested samples of moisture content. The test results were compared: sample $1 \#$ moisture content is 2.67 times sample 0\#, sample 2\# moisture content is 1.6 times sample 0\#, sample 3 \# moisture content is 1.49 times sample 0\#. The water content in silicone oil increased, which not only increases polar molecule, resulting in dielectric properties decrease, andalso catalyzes silicone accelerated aging, which is shown in Figure 5.

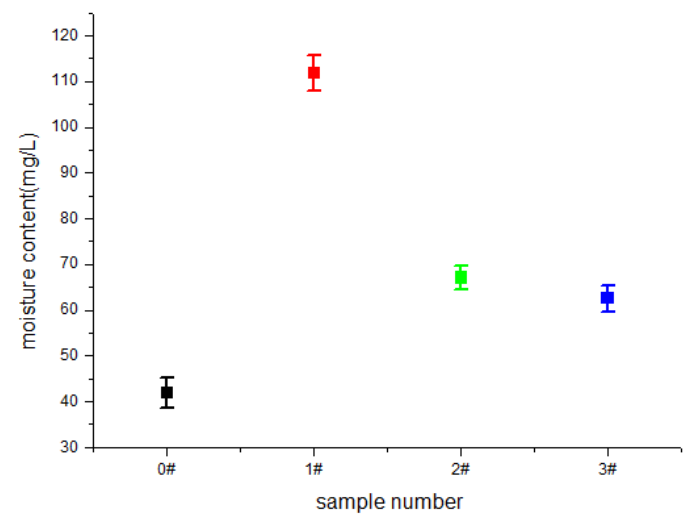

Figure 5. Moisture content of samples

\section{CONCLUSION}

Based on the Aging Characteristics of high-voltage silicone oil-filled cable termination, infrared imaging, infrared spectroscopy, dielectric properties, and moisture content tests can detect and react silicone oil aging characteristics inside cable termination from different angles, so they are important methods for detecting silicone oil aging characteristics.

Infrared imaging is a convenient application technology on site, and the detection result is more obvious and intuitive, able to reflect material changes in the macroscopic properties.

FTIR analysis can identify each group of silicone oil by corresponding characteristic absorption peaks and reflecting the silicone oil aging characteristics clearly. Silicone oil aging characteristic peaks is the $\mathrm{PH}$ bond stretching vibration near $2360 \mathrm{~cm}^{-1}$. There is not effect of $\mathrm{Si}-\mathrm{CH}_{3}$ bond, $-\mathrm{CH}_{3}$ bond and $\mathrm{Si}-\mathrm{O}-\mathrm{Si}$ bond before and after aging. 
Compared with $30^{\circ} \mathrm{C}$, the dielectric properties test of silicone oil better reflect the differences performance changes at $90^{\circ} \mathrm{C}$. Changes in the dielectric properties of silicone oil are mainly caused by the presence of water molecules and the degradation of polymer materials. The moisture content test can intuitively reflect the content of the water molecules in silicone oil is to assess if the aging performance of silicone oil has certain advantages.

\section{ACKNOWLEDGEMENTS}

The research reported in this work was kindly supported by a grant from Technology Project Foundation of China Southern Power Grid Company (K-GX2013-013).

\section{REFERENCES}

[1] Li XinPing, Liu Shougong \& Cao Xiaolong, et al. 2002. Development in the design of HV cable termination. Electric Wire and Cable, (3): 11-14.

[2] T. Ishikawa, K. Yasuda, S. Okabe S, Kaneko \& S Yanabu. Streaming Electrification Characteristics of Silicone Oil with Oil Temperature Increase.

[3] H. Yoshida. \& S. Yanabu, 2003. The Breakdown Characteristics of the Silicone Oil for Electric Power Apparatus, ISH2003, Delft, Netherlands, August 25-29.

[4] T.C. Champion, F. \& Marciano Agostinelli, R.D. 1995. Rosevear, Long term pre-qualification testing program on a $230 \mathrm{kV}$ XLPE cable system, IEEE Trans. POW. Del., 10: 10-17.

[5] T. Suzuki, S. Murakami. \& S. Kobayashi. 1983. Decomposition products of silicon liquid due to electric discharge, IEEE Trans. Electr. Insul., 18: 152-157.

[6] Sihui Hu, Ruzhang Wang, Qichao Song \& Yadong Li. 2006. The PD Characteristics of composite insulation for Polytetrafluoroethylene membrane silicone. High Voltage apparatus. (5):72-76.

[7] Liu Gang, Liu Yigang, Wang Chao \& Yi Zepei. The study on evaluation model for Prefabricated HV stress cone of cable Terminations weakness based on finite element. Conference Record of the 2006 IEEE International Symposium on Electrical Insulation. pp: 486-489.

[8] Liland K B, Bjorke, A. Kornberg, Hvidstens Faremo H. \& Bjerkan E. 2009. Failure Modes and Condition Assessment of High Voltage Oil Filled XLPE Terminations. 29th Electrical Insulation Conference Montreal, CANADA, MAY31-JUN03.

[9] Chen Chengwei, Liu Gang \& Li Jian, et al. The Study on Evaluation Model for Water in Silicone Oil of Cable Termination and Simulate Experiment. Conference Record of the 2008 IEEE International Symposium on Electrical Insulation. pp: 712-715.

[10]Wang Yanwei, Wei Chengwen \& Dai Jingxu. 2004 Study on mechanism of abnormal heat for high voltage cable end of Rolling 1344 line. High Voltage Engineering. 30(136): 65-98.
[11]Luo Zhenhai, Lu Guojun \& Wang Xiaobing. 2007. Study on fever cause of Porcelain sets High Voltage Cable Terminal. High Voltage Engineering. 33(11): 240-244.

[12] Jliang Shen, Junhua Luo \& Mei Tang. 2005. Analysis of Explosion Accident of $110 \mathrm{kV}$ Power Cable Termination. High Voltage Apparatus. 31(11): 74-75.

[13]WG B1. 29 Guidelines for maintaining the integrity of XLPE cable accessories.

[14]Fei Liang, MacAlpine Mark \& Zhicheng Guan. 2014. Formation of Dry-band in the Process of Pollution Flashover Based on Infrared Thermal Image Analysis. High Voltage Engineering. 40(1): 138-146.

[15] Jin Shao, Wuyan Hu \& Fengming Jia. 2013. Application of infrared thermal imaging technology to condition-based maintenance of power equipment. High Voltage apparatus. 49(1): 126-133.

[16]Hongying He, Yingjian Yang, Jiangang Yao, Zhenglong Jiang \& Yanping Qian. 2010. Influence Factors Research of Insulator Contamination Grades Detection Based on Infrared Image. High Voltage Engineering, (36): 1730-1737.

[17]Xingliang Jiang \& Qiangfeng Xia. 2010. Influence of Contamination to Infrared Imaging Method Detecting Deterioration Insulators. High Voltage Engineering, (36):1619-1625.

[18] Yunsheng Wang. 2004. Diagnoses of overheat defectiveness of the cable head by infrared technique, High Voltage Engineering. (16): 24-28

[19]Baoshu Li, Xiaohui Zhu, Shutao Zhao \& Wendong Niu. 2006. HV Power Equipment Diagnosis Based on Infrared Imaging Analyzing. 2006 International Conference on Power System Technology, 1-4.

[20]Z. B. Zhao, Q. Gao \& R. Li. 2005. Realization of IR temperature measurement in substation remote video monitoring system. Telecommunications for Electric Power System, China, (26): 27-32.

[21]Huangqiang Li \& Yunlian Sun. 2009. Research on HV-Power Equipment Diagnosis by Infrared Image Edge Detection. Power and Energy Engineering Conference, pp: 1-4.

[22]Baoshu Li, Xiaohui Zhu, Shutao Zhao \& Wendong Niu. HV Power Equipment Diagnosis Based on Infrared Imaging Analyzing. 2006 International Conference on Power System Technology, pp: 1-4.

[23] Xiaojuan Wu, Zhifang Cai, Qiang Yuan. 2007. Analysis of the cause abnormal heating cable terminal. Guangdong Electric Power. 20(9): 40-42

[24]FU Jian. 2005. Infrared diagnosis for voltage-caused thermal faults of equipment. East China Electric Power, 3(35): 66-68.

[25]Zhuo Jinyu. Power Cable Structure Design.

[26]Ding Yang. The Synthesis and Application of new phosphorus-containing silicone flame retardant, Shaanxi University of Science and Technology, Master Thesis.

[27]Zhao Mingxing. 2007. The New Synthesis Process for Polydimethylsiloxane Zhengzhou University. 5. 